\title{
Predictors of complications from stoma closure in elective colorectal surgery: an assessment from the American College of Surgeons National Surgical Quality Improvement Program (ACSNSQIP)
}

\author{
T. R. de Paula ${ }^{1}$ S. Nemeth ${ }^{2}$ R. P. Kiran ${ }^{1}$ • D. S. Keller ${ }^{1,3}$ \\ Received: 19 April 2020 / Accepted: 13 July 2020 / Published online: 22 July 2020 \\ (c) Springer Nature Switzerland AG 2020
}

\begin{abstract}
Background There is significant morbidity after diverting ileostomy closure, so identifying predictors of complications could be of great benefit. The aim of our study was to evaluate the incidence and risk factors for postoperative morbidity after elective ileostomy closure.

Methods The ACS-NSQIP dataset was evaluated for elective ileostomy closures from 1/1/2015 to 12/31/2016. Demographic characteristics, operative, and postoperative outcomes were evaluated. The primary outcome was 30-day major morbidity (Clavien class III and greater). Secondary outcomes were rates and predictors of major morbidity, superficial site infection (SSI), reoperation, and readmission from multivariate logistic regression modeling.

Results We retrospectively evaluated 1885 patients. The median operative time was 65 (IQR 50-90) minutes and median length of stay was 3 (IQR 2-5) days. Major morbidity was recorded in 6.7\%, including mortality (1.0\%), deep/organ space SSI (2.6\%), dehiscence (0.8\%), reintubation ( $0.5 \%)$, sepsis (1.7\%), septic shock (0.8\%), and reoperation (3.7\%). Readmission was recorded in $9.7 \%$ and $6.2 \%$ had SSI. Multivariate logistic regression showed male sex (OR 1.584; 95\% CI 1.068-2.347; $p=0.022$ ) and longer operative time (OR 1.004; 95\% CI 1.001-1.007; $p=0.009$ ) were among those variables associated with increased odds of major morbidity. Dyspnea (OR 2.431; 95\% CI 1.139-5.094; $p=0.021$ ) and longer operative time (OR 1.003; 95\% CI 1.001-1.007; $p=0.034$ ) were among the independent risk factors for SSI. Male sex (OR 2.246; 95\% CI 1.297-3.892; $p=0.004$, chronic obstructive pulmonary disease (OR 2.959; 95\% CI 1.153-7.591; $p=0.024$ ), and longer operative time (OR 1.005; 95\% CI 1.001-1.009; $p=0.011$ ) were associated with increased odds of reoperation. Chronic obstructive pulmonary disease (OR 2.578; 95\% CI 1.338-4.968; $p=0.005$ ), wound infection (OR 2.680; 95\% CI 1.043-6.890; $p=0.041$ ), and inflammatory bowel disease (OR 2.565; 95\% CI 1.203-5.463; $p=0.015$ ) were associated with increased odds of readmission.

Conclusions Elective stoma closure has significant risk of morbidity. Patients with longer operative times were at increased risk for major morbidity, overall SSI, and reoperation. From the analysis, factors specifically associated with major morbidity, overall infectious complications, readmissions, and reoperations were identified. This information can be used to prospectively prepare for these high-risk patients, potentially improving postoperative outcomes.
\end{abstract}

Keywords National surgical quality improvement program (NSQIP) · Colorectal surgery $\cdot$ Patient readmission $\cdot$ Morbidity · Reoperation $\cdot$ Surgical outcomes $\cdot$ Ileostomy $\cdot$ Operative time $\cdot$ Risk factors

\section{Introduction}

Electronic supplementary material The online version of this article (https://doi.org/10.1007/s10151-020-02307-5) contains supplementary material, which is available to authorized users.

D. S. Keller

debby_keller@hotmail.com

Extended author information available on the last page of the article
An anastomotic leak is one of the most feared complications after colorectal surgery. Despite advances in instrumentation, technology, and surgical technique, the rate of anastomotic leaks remains steady; it is reported in up to $20 \%$ of resections [1, 2]. The etiology of anastomotic leaks is multifactorial and their impact is extensive, adding considerable morbidity, mortality, increased cancer recurrence rates, and 
a substantial economic burden [2-8]. Fecal diversion with a defunctioning or loop ileostomy is often used in colorectal surgery to protect a distal colonic anastomosis and mitigate the sequelae of anastomotic leak [9]. Thus, temporary diversion can serve an important functional purpose and the temporary ileostomies are generally closed.

The subsequent diverting ileostomy closure is often thought of as a simple and safe procedure. However, many studies have demonstrated high morbidity rates following diverting ileostomy closure, with substantial impact on the patient and healthcare system [10]. There are differences in the definition of complications, but morbidity rates after stoma closure vary from 10 to $48.2 \%$ [11-13]. Commonly encountered complications include small bowel obstruction, wound infection, stoma site hernias, anastomotic leak, and enterocutaneous fistulae, with the possible need for further reoperation, and its associated operative mortality, inpatient stay, and costs [12]. This single procedure accounts for a disproportionate share of morbidity, mortality, and excess hospital days [14]. Although the frequency of severe postoperative complications after ileostomy closure is substantial, there is little data regarding risk factors for the complications after surgery.

With the large burden of stoma closure-related morbidity, identifying predictors of complications could serve great benefit for patients and providers.

Our goal was to evaluate the postoperative outcomes and identify preoperative variables associated with complications after diverting loop ileostomy closure. Our hypothesis was that there are variables that can be identified prospectively, potentially improving postoperative outcomes in these high-risk patients.

\section{Materials and methods}

A review of the American College of Surgeons National Surgical Quality Improvement Program (ACS-NSQIP) participant user file (PUF) was performed to identify patients who had an ileostomy closure between 1/1/2015 and12/31/2016. The PUF contains patient level, aggregate data on cases submitted to the national validated, risk-adjusted, outcomesbased quality improvement program. Stoma closure cases were recognized by Current Procedural Terminology (CPT) code 44620. Patients were included if over 18 years of age, had no concurrent procedures, and the procedure was performed on an elective basis. Patients were excluded if their postoperative diagnosis included colostomy.

Preoperative patient baseline demographic and clinical characteristics as well as operative and postoperative outcomes, especially pertaining to 30-day morbidity and mortality, were evaluated.
The primary outcome was major morbidity comprising Clavien class $\geq$ III complications, the need for reintervention and reoperation, and 30-day postoperative mortality. Secondary endpoints included overall surgical site infection (SSI) comprised of superficial, deep, and/or organ space SSI, reoperation, and readmission after ileostomy closure.

Data definitions were compliant with the ACS_NSQIP Data Dictionary (available online at: https://site.acsnsqip. org/wp-content/uploads/2012/03/ACS-NSQIP-Participan t-User-Data-File-User-Guide_06.pdf). The handling of missing data is outlined in Appendix 1. Variables with $<1 \%$ missing were imputed to the median/mode. White blood cell (WBC) count and hematocrit (HCT) had 8-10\% missing data and were both imputed via random forest multiple imputation.

Patients with an operative time of less than 10 min were excluded due to a probable error in data entry. Surgical indication was determined through grouping the postoperative diagnoses (ICD-9 and 10) which is outlined in Appendix 2. Some complications have an additional field option of present at time of surgery (PATOS). The following fields were only considered to be a complication if they were marked yes to the complication and it was not PATOS: superficial SSI, deep incisional SSI, organ space SSI, pneumonia, urinary tract infection, sepsis, and septic shock.

For statistical analysis, data were expressed as frequencies and percentages for categorical variables. Continuous variables are expressed as either mean (SD) or median (IQR) depending on normality which was tested via QQ Plots. Next, multiple imputation via random forest was performed for both HCT and WBC. Five iterations of each missing data point were imputed within five different imputations for convergence and thus, the univariable logistic regression results for these two variables are pooled results based on multiple iterations of data. Univariable logistic regression was performed for all preoperative risk factor variables available in the database (except for preoperative transfusion, ventilator dependence, renal failure, and congestive heart failure which were excluded as they did not have enough instances), as well as hematocrit and white blood cell count lab values, and operative time (Table 1). Significant variables were then included in a multivariable model along with sex, age, race, and surgical indication. Variables in the model were checked for collinearity using the Variance Inflation Factor (VIF). For each regression, a $p$ value $<0.05$ was considered to be significant. R statistical software (version 3.3.3, R Foundation) was used for statistical analyses.

\section{Ethical statement}

This study was approved by the Columbia University Institutional Review Board (Protocol \#AAAS 1001) with waiver of consent for Research of Existing Data/Records/Specimens 
Table 1 Patient characteristics, disease factors and intervention $(N=1885)$

\begin{tabular}{llll}
\hline Variable & $N(\%)$ median (IQR) & Variable & $N(\%)$ median (IQR) \\
\hline Male sex & $060(56.2 \%)$ & Sepsis & $4(0.7 \%)$ \\
Age, years & $57.0[47.0-66.0]$ & HCT (unit) & $38.6[35.4-41.9]$ \\
Race & & WBC count (unit) & $6.6[5.2-8.3]$ \\
White & $1378(73.1 \%)$ & Wound class & \\
Black & $121(6.4 \%)$ & 1 & $30(1.6 \%)$ \\
Asian & $54(2.9 \%)$ & 2 & $1549(82.2 \%)$ \\
Other & $332(17.6 \%)$ & 3 & $251(13.3 \%)$ \\
BMI, kg/m & $25.9[22.8-29.9]$ & 4 & $55(2.9 \%)$ \\
Chemotherapy & $526(27.9 \%)$ & ASA class & \\
Diabetes mellitus & $182(9.7 \%)$ & 1 & $30(1.6 \%)$ \\
Smoking & $367(19.5 \%)$ & 2 & $1026(54.4 \%)$ \\
Dyspnea & $77(4.1 \%)$ & 3 & $797(42.3 \%)$ \\
COPD & $64(3.4 \%)$ & 4 & $32(1.7 \%)$ \\
CHF & $5(0.3 \%)$ & Functional status & $22(1.2 \%)$ \\
Hypertension meds & $628(33.3 \%)$ & Transfer patient & $17(0.9 \%)$ \\
Renal failure & $3(0.2 \%)$ & Operative time (min) & $65.0[50.0-90.0]$ \\
Dialysis & $13(0.7 \%)$ & Surgical indication & \\
Disseminated cancer & $113(6.0 \%)$ & Attention to ileostomy & $1489(79.0 \%)$ \\
Wound infection & $28(1.5 \%)$ & Rectal cancer & $117(6.2 \%)$ \\
Ventilator dependence & $2(0.1 \%)$ & Diverticular disease & $50(2.7 \%)$ \\
Weight loss & $83(4.4 \%)$ & IBD & $44(2.3 \%)$ \\
Bleeding disorder & $64(3.4 \%)$ & Other & $166(8.8 \%)$ \\
Preoperative transfusion & $2(0.1 \%)$ & Colon cancer & $19(1.0 \%)$ \\
Steroid use & $132(7.0 \%)$ & Length of stay (days) & $3.0[2.0-5.0]$ \\
\hline Pros & & \\
\hline
\end{tabular}

Post-single imputation

$B M I$ body mass index, $C O P D$ chronic obstructive pulmonary disease, $C H F$ chronic heart failure, $H C T$ hematocrit; at time of surgery, $W B C$ white blood cell (WBC count at time of surgery), IBD inflammatory bowel disease, ASA American Society of Anesthesiologists (46.101(b) 4). This study was performed in compliance with The Strengthening the Reporting of Observational Studies in Epidemiology (STROBE) checklist for reporting observational studies.

\section{Results}

During the study period, 1,885 patients had elective ileostomy closure, met inclusion criteria and were included in the analysis. In the cohort, the median age was 57 years (range 47-66 years) [47, 67], and 56.2\% were male. The median body mass index (BMI) was $25.9 \mathrm{~kg} / \mathrm{m}^{2}$ (range $22.8-29.9 \mathrm{~kg} / \mathrm{m}^{2}$ ), and $56.0 \%$ were American Society of Anesthesiologists (ASA) class 1-2. Prior to surgery, 27.9\% (526) had chemotherapy and 7.0\% (132) were on steroids. The median operative time was $65 \mathrm{~min}$ (range 50-90 min), and overall median hospital length of stay (LOS) was 3 days (range 2-5 days).

Full patient demographics are shown in Table 1.
Postoperatively, the overall morbidity was $20.3 \%$ $(n=388)$. Major morbidity was experienced in $126(6.7 \%)$ patients, including mortality $(n=18,1.0 \%)$, deep/organ space SSI requiring intervention $(n=49,2.6 \%)$, reintubation $(\mathrm{n}=9,0.5 \%)$, sepsis $(n=32,1.7 \%)$, septic shock $(n=15$, $0.8 \%)$, and unplanned reoperation $(n=70,3.7 \%)$. Within 30 days of surgery, $6.2 \%$ had overall SSI and $9.7 \%$ were readmitted. The observed venous thromboembolism rate was low, with $0.2 \%$ and $0.7 \%$ of patients suffering pulmonary embolism and deep vein thrombosis, respectively.

Details of the postoperative complications are displayed in Table 2. While the overall median hospital LOS was 3 days, in patients with any complication, the median hospital LOS was 5 days [3-10], and in patients with major morbidity, the median LOS was 8 days $[3,13]$.

On multivariate logistic regression, male sex and longer operative time were associated with higher odds of major morbidity (Table 3). The odds ratio (OR) for each additional minute of operating room time indicated that 15 extra minutes in the operating room equates to a $6 \%$ 
Table 230 day morbidity and mortality following stoma closure $(N=1885)$

\begin{tabular}{lrlr}
\hline Variable & $N(\%)$ & Variable & $N(\%)$ \\
\hline Mortality & $18(1.0 \%)$ & Renal insufficiency & $5(0.3 \%)$ \\
Overall morbidity & $388(20.3 \%)$ & Renal failure & $5(0.3 \%)$ \\
Major morbidity & $126(6.7 \%)$ & Urinary tract infection & $18(1.0 \%)$ \\
Overall SSI & $116(6.2 \%)$ & Stroke/CVA & $4(0.2 \%)$ \\
Superficial SSI & $52(2.8 \%)$ & Cardiac arrest & $3(0.2 \%)$ \\
Deep SSI & $15(0.8 \%)$ & Myocardial infarction & $3(0.2 \%)$ \\
Organ space SSI & $49(2.6 \%)$ & Bleeding requiring transfusion & $26(1.4 \%)$ \\
Wound dehiscence & $16(0.8 \%)$ & Deep vein thrombosis & $13(0.7 \%)$ \\
Pneumonia & $23(1.2 \%)$ & Sepsis & $32(1.7 \%)$ \\
Reintubation & $9(0.5 \%)$ & Septic shock & $15(0.8 \%)$ \\
Pulmonary embolism & $4(0.2 \%)$ & Reoperation & $70(3.7 \%)$ \\
Prolonged ventilation & $8(0.4 \%)$ & Readmission & $183(9.7)$ \\
\hline
\end{tabular}

SSI surgical site infection, CVA cerebrovascular accident

Table 3 Major morbidity results

\begin{tabular}{|c|c|c|c|c|c|}
\hline & $\begin{array}{l}\text { No major morbidity } N \\
\text { (1759) }\end{array}$ & Major morbidity $N(126)$ & OR & $95 \% \mathrm{CI}$ & $P$ value \\
\hline Male sex & $977(55.5 \%)$ & $83(65.9 \%)$ & 1.584 & {$[1.068-2.347]$} & $0.022^{\mathrm{a}}$ \\
\hline Age, years ${ }^{\mathrm{a}}$ & $57.0[47.0-66.0]$ & $58.0[46.0-70.0]$ & 1.005 & [0.992-1.018] & 0.453 \\
\hline \multicolumn{6}{|l|}{ BMI, $\mathrm{kg} / \mathrm{m}^{2}$} \\
\hline $18.5-25$ (reference) & $688(39.1 \%)$ & $51(40.5 \%)$ & & & \\
\hline$<18$ & $70(4.0 \%)$ & $8(6.3 \%)$ & 1.556 & {$[0.682-3.550]$} & 0.293 \\
\hline $25-30$ & $573(32.6 \%)$ & $34(27.0 \%)$ & 0.726 & {$[0.458-1.153]$} & 0.174 \\
\hline $30+$ & $428(24.3 \%)$ & $33(26.2 \%)$ & 1.022 & [0.638-1.639] & 0.926 \\
\hline Dyspnea & $66(3.8 \%)$ & $11(8.7 \%)$ & 1.895 & {$[0.909-3.951]$} & 0.088 \\
\hline COPD & $54(3.1 \%)$ & $10(7.9 \%)$ & 2.125 & {$[0.964-4.683]$} & 0.062 \\
\hline \multicolumn{6}{|l|}{ Surgical indication } \\
\hline Attention to ileostomy (reference) & $1394(79.2 \%)$ & $95(75.4 \%)$ & & & \\
\hline Colon cancer & $18(1.0 \%)$ & $1(0.8 \%)$ & 0.729 & {$[0.095-5.607]$} & 0.761 \\
\hline Diverticular disease & $49(2.8 \%)$ & $1(0.8 \%)$ & 0.255 & [0.034-1.917] & 0.184 \\
\hline IBD & $40(2.3 \%)$ & $4(3.2 \%)$ & 1.692 & {$[0.570-5.028]$} & 0.343 \\
\hline Other & $151(8.6 \%)$ & $15(11.9 \%)$ & 1.209 & {$[0.665-2.201]$} & 0.533 \\
\hline Rectal cancer & $107(6.1 \%)$ & $10(7.9 \%)$ & 1.323 & {$[0.662-2.643]$} & 0.428 \\
\hline Operative time, minutes ${ }^{\mathrm{a}}$ & $65.0[49.0-90.0]$ & $74.0[56.0-97.8]$ & 1.004 & [1.001-1.007] & $0.009^{\mathrm{a}}$ \\
\hline
\end{tabular}

$B M I$ body mass index, $C O P D$ chronic obstructive pulmonary disease, $I B D$ inflammatory bowel disease, $H C T$ hematocrit, $W B C$ white blood cell ${ }^{a}$ Median, [range]

increase in the odds of major morbidity. The regression model showed that dyspnea and longer operative time were associated with higher odds of overall SSI (Table 4). Male sex, chronic obstructive pulmonary disease (COPD), and longer operative time were significantly independently associated with higher odds of reoperation (Table 5). COPD, wound infection, and inflammatory bowel disease (IBD) as the surgical indication were all independently associated with higher odds of readmission (Table 6).

\section{Discussion}

In this national review of ileostomy closure procedures, over $20 \%$ of patients had complication reported overall, with nearly $10 \%$ experiencing major complications and unplanned readmission. We found there are variables that can be identified prospectively, including male sex, IBD diagnosis, and comorbidities of COPD and dyspnea, as well as those related to the procedure, in longer operative 
Table 4 Overall SSI results

\begin{tabular}{|c|c|c|c|c|c|}
\hline & No overall SSI $N$ (1769) & Overall SSI $N(116)$ & OR & $95 \% \mathrm{CI}$ & $P$ value \\
\hline Male sex & $1002(56.5 \%)$ & $58(51.8 \%)$ & 0.834 & {$[0.550-1.208]$} & 0.309 \\
\hline Age, years ${ }^{\mathrm{a}}$ & $57.0[47.0-66.0]$ & $55.0[40.8-65.0]$ & 0.913 & {$[0.975-1.001]$} & 0.062 \\
\hline \multicolumn{6}{|l|}{ BMI, $\mathrm{kg} / \mathrm{m}^{2}$} \\
\hline $18.5-25$ (reference) & $698(39.4 \%)$ & $41(36.6 \%)$ & & & \\
\hline$<18$ & $75(4.2 \%)$ & $3(2.7 \%)$ & 0.608 & {$[0.198-2.230]$} & 0.508 \\
\hline $25-30$ & $579(32.7 \%)$ & $28(25.0 \%)$ & 0.809 & {$[0.512-1.411]$} & 0.529 \\
\hline $30+$ & $421(23.7 \%)$ & $40(35.7 \%)$ & 1.514 & {$[0.966-2.460]$} & 0.070 \\
\hline Dyspnea & $68(3.8 \%)$ & $9(8.0 \%)$ & 2.431 & {$[1.139-5.094]$} & $0.021^{\mathrm{a}}$ \\
\hline \multicolumn{6}{|l|}{ Surgical Indication } \\
\hline Attention to ileostomy (reference) & $1406(79.3 \%)$ & $83(74.1 \%)$ & & & \\
\hline Colon cancer & $17(1.0 \%)$ & $2(1.8 \%)$ & 1.741 & {$[0.383-7.877]$} & 0.475 \\
\hline Diverticular disease & $48(2.7 \%)$ & $2(1.8 \%)$ & 0.623 & {$[0.149-2.740]$} & 0.547 \\
\hline IBD & $40(2.3 \%)$ & $4(3.6 \%)$ & 1.414 & {$[0.483-4.233]$} & 0.518 \\
\hline Other & $152(8.6 \%)$ & $14(12.5 \%)$ & 1.200 & [0.696-2.389] & 0.418 \\
\hline Rectal cancer & $110(6.2 \%)$ & $7(6.2 \%)$ & 1.104 & {$[0.491-2.472]$} & 0.815 \\
\hline Operative time, minutes ${ }^{\mathrm{a}}$ & $65.0[49.0-89.0]$ & $75.5[56.0-113.3]$ & 1.003 & [1.001-1.007] & $0.034^{\mathrm{a}}$ \\
\hline
\end{tabular}

$B M I$ body mass index, $C O P D$ chronic obstructive pulmonary disease, IBD inflammatory bowel disease, $H C T$ hematocrit, SSI surgical site infection

${ }^{a}$ Median [range]

Table 5 Reoperation results

\begin{tabular}{|c|c|c|c|c|c|}
\hline & $\begin{array}{l}\text { No reop } \\
N(1815)\end{array}$ & $\begin{array}{l}\text { Reop } \\
N(70)\end{array}$ & OR & $95 \% \mathrm{CI}$ & $P$ value \\
\hline Male Sex & $1,009(55.6 \%)$ & $51(72.9 \%)$ & 2.246 & [1.297-3.892] & 0.004 \\
\hline Age, years ${ }^{\mathrm{a}}$ & $57.0[47.0-66.0]$ & $58.0[45.3-67.0]$ & 1.000 & {$[0.983-1.016]$} & 0.971 \\
\hline \multicolumn{6}{|l|}{ BMI kg/m² } \\
\hline 18.5-25 (reference) & $707(39.0 \%)$ & $32(45.7 \%)$ & & & \\
\hline$<18$ & $74(4.1 \%)$ & $4(5.7 \%)$ & 1.477 & {$[0.482-4.522]$} & 0.494 \\
\hline $25-30$ & $590(32.5 \%)$ & $17(24.3 \%)$ & 0.563 & {$[0.304-1.042]$} & 0.067 \\
\hline $30+$ & $444(24.5 \%)$ & $17(24.3 \%)$ & 0.824 & {$[0.441-1.542]$} & 0.545 \\
\hline COPD & $58(3.2 \%)$ & $6(8.6 \%)$ & 2.959 & {$[1.153-7.591]$} & 0.024 \\
\hline \multicolumn{6}{|l|}{ Surgical indication } \\
\hline Attention to ileostomy (reference) & $1,436(79.1 \%)$ & $53(75.7 \%)$ & & & \\
\hline Colon cancer & $18(1.0 \%)$ & $1(1.4 \%)$ & 1.391 & {$[0.178-10.892]$} & 0.753 \\
\hline Diverticular disease & $50(2.8 \%)$ & $0(0.0 \%)$ & 0.000 & {$[0.000-\mathrm{NA}]$} & 0.979 \\
\hline IBD & $40(2.2 \%)$ & $4(5.7 \%)$ & 2.855 & [0.929-8.776] & 0.067 \\
\hline Other & $161(8.9 \%)$ & $5(7.1 \%)$ & 0.683 & {$[0.256-1.826]$} & 0.447 \\
\hline Rectal cancer & $110(6.1 \%)$ & $7(10.0 \%)$ & 1.631 & {$[0.713-3.728]$} & 0.246 \\
\hline Operative time, minutes ${ }^{\mathrm{a}}$ & $65.0[50.0-90.0]$ & 71.5 [54.3-96.3] & 1.005 & {$[1.001-1.009$} & 0.011 \\
\hline
\end{tabular}

$B M I$ body mass index; $C O P D$ chronic obstructive pulmonary disease, $I B D$ inflammatory bowel disease, $H C T$ hematocrit; $W B C$ white blood cell ${ }^{a}$ Median, [range]

times. Based on these results, the non-modifiable variables can be used for risk-stratification of higher-risks patients, and the modifiable factors used as metrics for quality improvement in these cases.
Patients who have a diverting ileostomy created are inherently at higher risk for complications. These patients are in general sicker, with more serious diagnoses such as cancer, IBD, and complicated diverticulitis where they can be 
Table 6 Readmission results

\begin{tabular}{llllll}
\hline & No readmit $N(1702)$ & Readmit $N(183)$ & OR & $95 \%$ CI & $P$ value \\
\hline Male sex & $959(56.3 \%)$ & $101(55.2 \%)$ & 1.013 & {$[0.739-1.390]$} & 0.936 \\
Age, years ${ }^{\mathrm{a}}$ & $57.0[47.0-66.0]$ & $57.0[44.0-68.0]$ & 0.998 & {$[0.988-1.009]$} & 0.748 \\
BMI, kg/m ${ }^{2}$ & & & & & \\
$18.5-25($ reference $)$ & $657(38.6 \%)$ & $82(44.8 \%)$ & & & \\
$<18$ & $70(4.1 \%)$ & $8(4.4 \%)$ & 0.880 & {$[0.401-1.931]$} & 0.750 \\
$25-30$ & $557(32.7 \%)$ & $50(27.3 \%)$ & 0.737 & {$[0.504-1.079]$} & 0.116 \\
$30+$ & $418(24.6 \%)$ & $43(23.5 \%)$ & 0.858 & {$[0.576-1.280]$} & 0.454 \\
& & & 2.578 & {$[1.338-4.968]$} & 0.005 \\
& & & 2.680 & {$[1.043-6.890]$} & 0.041 \\
Surgical indication & & & & & \\
Attention to ileostomy & $1,344(79.0 \%)$ & $145(79.2 \%)$ & & & \\
Colon cancer & $18(1.1 \%)$ & $1(0.5 \%)$ & 0.553 & {$[0.073-4.212]$} & 0.567 \\
Diverticular disease & $47(2.8 \%)$ & $3(1.6 \%)$ & 0.520 & {$[0.157-1.721]$} & 0.284 \\
IBD & $34(2.0 \%)$ & $10(5.5 \%)$ & 2.565 & {$[1.203-5.463]$} & 0.015 \\
Other & $150(8.8 \%)$ & $16(8.7 \%)$ & 0.941 & {$[0.541-1.639]$} & 0.830 \\
Rectal cancer & $109(6.4 \%)$ & $8(4.4 \%)$ & 0.678 & {$[0.321-1.435]$} & 0.310 \\
Operative time, minutes ${ }^{\mathrm{a}}$ & $65.0[49.0-90.0]$ & $67.0[53.5-89.0]$ & 1.001 & {$[0.997-1.004]$} & 0.657 \\
\hline
\end{tabular}

$B M I$ body mass index, $C O P D$ chronic obstructive pulmonary disease, $I B D$ inflammatory bowel disease, $H C T$ hematocrit, $W B C$ white blood cell

${ }^{a}$ Median [range]

malnourished and physiologically depleted. This group commonly has greater comorbidities, including steroid use and receipt of radiation and chemotherapy and are more complex cases, with higher risks of anastomotic leak and sepsis, these qualities are why a diverting ileostomy was created. Some of these risks may still be pertinent at the time of ileostomy closure, and the consequences of stoma reversal are thus often underestimated [10].

The decision to close an ileostomy is complex, and must take into account the patient's wishes, fitness, comorbidities, diagnosis and adjuvant treatments, as well as the surgeon's perspective. When the decision to close the ileostomy is made, all efforts should be focused on methods to reduce complications in these high-risk patients. Factors such as the timing of closure and impact of distal limb irrigation may be considered $[15,16]$. To date, there are conflicting results on the optimal interval from creation to closure on complications [17-20] or the impact of distal limb irrigation $[15,16]$. In the meantime, using the variables identified here for proactively recognizing high-risk patients and putting the information into action proactively may help reduce major morbidity, SSI, readmissions, and reoperations after surgery.

Aligned with our findings, prior studies have described the overall complication rate of diverting ileostomy closure between 10 and $30 \%$, with a $10-15 \%$ readmission rate $[15$, 20-22]. While substantial, these complications are often underestimated [10]. Our work is the first to examine major morbidity specifically, which is more likely to impact quality outcomes metrics and have longer and costlier index and readmission episodes [23, 24]. Major complications in our national sample of ileostomy reversals were $6.7 \%$, and major complication patients had longer LOS. a median of 8 days compared to 5 days with any complication, and 3 days overall. Our results agree with prior work that showed that regardless of the complication class, LOS is prolonged when complications occur [22]. With these results, there is great potential for cost savings with reducing complications after ileostomy closure.

Published work has shown that complications both before and after discharge most often stem from preventable issues, such as wound infection [21, 22]. This agrees with our findings with SSI being the most common complication. The increased use of SSI bundles with enhanced recovery programs, clean closure trays in the operating room, closure using the purse-string technique compared to linear skin closure, and post-discharge monitoring using mHealth apps could impact this complication [25, 26]. Furthermore, most surgical readmissions occur before the first scheduled follow-up [21, 23]. Applying our results, patients at high-risk for major morbidity, SSI, reoperation and readmission can be prospectively identified, which may allow for modifications to the standard care pathway to improve outcomes in this group. We found an association of preoperative dyspnea with SSI and COPD with reoperation rates. Pre-habilitation, which improves functional capacity for surgery, and helps mitigate the negative impact from frailty and comorbidities on outcomes, could be applied in these ileostomy closure patients. Pre-habilitation has been proven to reduce 
postoperative complications, readmissions, and costs of care in high-risk patients having elective abdominal surgery, primarily from increased aerobic capacity $[27,28]$. These benefits could be extended to this cohort, who likely are not considered for pre- habilitation, as the practice is not routine in the United States or may be considered unnecessary for a diverting loop ileostomy closure.

We saw the independent association of longer operative times with increases in major morbidity, overall SSI, and reoperation. Earlier work has shown that longer operative times as well as intraoperative complications, intensive care unit care, longer LOS, and skilled nursing at discharge were predictive of readmission after ileostomy reversal [29]. However, none of these variables can be prospectively applied for risk stratification. Our work adds to the published literature by showing the independent relationship of operative time with major postoperative morbidity, SSI, and unplanned reoperation. Longer operative time may be a surrogate for patient factors (such as obesity or frailty), increased case complexity, intraoperative events, or surgeon experience. Longer operative time for ileostomy reversal while only one indicator, might be associated with modifiable and nonmodifiable factors that can interplay and potentiate the negative risks on postoperative outcomes and thus it is important indicator to help identify patients at increased risk before the complications occur. Complexity of cases and intraoperative events are mostly non-modifiable factors, while for surgeon's expertise, there has been support for regionalization of care to specialty centers to mitigate the disparities and improve outcomes [30-33]. The same planning could be applied to ileostomy reversal cases, where patients identified as highrisk are directed to specialty care centers that are better equipped to manage higher complexity patients. Equally, low risk cases could be identified for same day discharge after reversal, so reducing average LOS [34].

We recognize the limitations in this work. The ACS NSQIP is an administrative data source with the inherent risks of miscoding and data entry errors, including coding for colostomy creation instead of ileostomy creation. However, we would expect the impact of such errors to have a minor impact in this large sample size. Prior work has shown the original indication for the ileostomy may impact outcomes after the ileostomy closure [17]. However, the majority of patients evaluated had the presence of the ileostomy, not the diagnosis at the index operation as their diagnosis code, which creates an inherent limitation in this analysis. Additionally, the reporting of postoperative complications might be underestimated in this work, as rates of postoperative ileus and obstruction not requiring operation are not specifically reported. There is also the risk of confounding of the variables of interest, which we attempted to overcome with the adjusted regression models. The readmission outcome is all-comer/all-cause, and patients may be readmitted for an independent comorbidity unrelated to the stoma closure.

Despite any limitations, there are important clinical implications from this work. With the individual variables that increase the risks of major morbidity, reoperation, and readmission prospectively identified, pre-habilitation can be initiated routinely in high-risk cases, process changes can be made to standard care pathways during the impatient stay, and disposition planning prospectively commenced to prepare for the common complications recognized in this population after surgery. In addition, changes can be made pre- and intraoperatively using the ACS NSQIP risk calculator from provided NSQIP demographic fields, which can aid in risk stratification and the consent process [35].

\section{Conclusions}

This national evaluation of ileostomy closure found overall complications and major complications are common, occurring in $20 \%$ and $10 \%$ of cases, respectively. From the analysis, factors specifically associated with major morbidity, overall infectious complications, readmissions, and reoperations were identified. Specifically, patients with longer operative times were at increased risk for major morbidity, overall SSI, and reoperation. This information can be used to prospectively prepare for these high-risk patients, potentially improving postoperative outcomes and healthcare utilization. A prospective study is underway to apply and validate these findings and assess their impact on outcomes after ileostomy closure.

Funding The authors received no financial support or funding for this work.

\section{Compliance with ethical standards}

Conflict of interest The authors have no conflicts of interest.

Ethical approval This study was approved by the Columbia University Institutional Review Board (Protocol \#AAAS1001) with waiver of consent for Research of Existing Data/Records/Specimens (46.101(b) 4). This study was performed in compliance with The Strengthening the Reporting of Observational Studies in Epidemiology (STROBE) checklist for reporting observational studies.

Informed consent Informed consent is not possible or required with this data source.

\section{References}

1. Vallance A, Wexner S, Berho M et al (2017) A collaborative review of the current concepts and challenges of anastomotic leaks in colorectal surgery. Colorect Dis 19:O1-O12 
2. Chadi SA, Fingerhut A, Berho M et al (2016) Emerging trends in the etiology, prevention, and treatment of gastrointestinal anastomotic leakage. J Gastrointest Surg 20:2035-2051

3. Bell SW, Walker KG, Rickard MJ et al (2003) Anastomotic leakage after curative anterior resection results in a higher prevalence of local recurrence. Br J Surg 90:1261-1266

4. Law WL, Choi HK, Lee YM, Ho JW, Seto CL (2007) Anastomotic leakage is associated with poor long-term outcome in patients after curative colorectal resection for malignancy. J Gastrointest Surg 11:8-15

5. Hyman NH, Osler T, Cataldo P, Burns EH, Shackford SR (2009) Anastomotic leaks after bowel resection: what does peer review teach us about the relationship to postoperative mortality. J Am Coll Surg 208:48-52

6. Hirst NA, Tiernan JP, Millner PA, Jayne DG (2014) Systematic review of methods to predict and detect anastomotic leakage in colorectal surgery. Colorect Dis 16:95-109

7. Hammond J, Lim S, Wan Y, Gao X, Patkar A (2014) The burden of gastrointestinal anastomotic leaks: an evaluation of clinical and economic outcomes. J Gastrointest Surg 18:1176-1185

8. Goto S, Hasegawa S, Hida K, Uozumi R, Kanemitsu Y, Watanabe T, Sugihara K, Sakai Y, Study Group for Nomogram of the JapaneseSociety for Cancer of the Colon and Rectum (2017) Multicenter analysis of impact of anastomotic leakage on long-term oncologic outcomes after curative resection of colon cancer. Surgery 162(2):317-324. https://doi.org/10.1016/j.surg.2017.03.005

9. Hanna MH, Vinci A, Pigazzi A (2015) Diverting ileostomy in colorectal surgery: when is it necessary. Langenbecks Arch Surg 400:145-152

10. Chow A, Tilney HS, Paraskeva P, Jeyarajah S, Zacharakis E, Purkayastha S (2009) The morbidity surrounding reversal of defunctioning ileostomies: a systematic review of 48 studies including 6,107 cases. Int J Colorect Dis 24:711-723

11. Pokorny H, Herkner H, Jakesz R, Herbst F (2005) Mortality and complications after stoma closure. Arch Surg 140:956-960 (discussion 960)

12. Kaidar-Person O, Person B, Wexner SD (2005) Complications of construction and closure of temporary loop ileostomy. J Am Coll Surg 201:759-773

13. Russek K, George JM, Zafar N, Cuevas-Estandia P, Franklin M (2011) Laparoscopic loop ileostomy reversal: reducing morbidity while improving functional outcomes. JSLS 15:475-479

14. Schilling PL, Dimick JB, Birkmeyer JD (2008) Prioritizing quality improvement in general surgery. J Am Coll Surg 207:698-704

15. Williams LA, Sagar PM, Finan PJ, Burke D (2008) The outcome of loop ileostomy closure: a prospective study. Colorect Dis 10:460-464

16. Garfinkle R, Trabulsi N, Morin N et al (2017) Study protocol evaluating the use of bowel stimulation before loop ileostomy closure to reduce postoperative ileus: a multicenter randomized controlled trial. Colorect Dis 19:1024-1029

17. Mansfield SD, Jensen C, Phair AS, Kelly OT, Kelly SB (2008) Complications of loop ileostomy closure: a retrospective cohort analysis of 123 patients. World J Surg 32:2101-2106

18. D'Haeninck A, Wolthuis AM, Penninckx F, D'Hondt M, D'Hoore A (2011) Morbidity after closure of a defunctioning loop ileostomy. Acta Chir Belg 111:136-141

19. Perez RO, Habr-Gama A, Seid VE et al (2006) Loop ileostomy morbidity: timing of closure matters. Dis Colon Rectum 49:1539-1545

20. Farag S, Rehman S, Sains P, Baig MK, Sajid MS (2017) Early vs delayed closure of loop defunctioning ileostomy in patients undergoing distal colorectal resections: an integrated systematic review and meta-analysis of published randomized controlled trials. Colorect Dis 19:1050-1057

21. Keller DS, Swendseid B, Khan S, Delaney CP (2014) Readmissions after ileostomy closure: cause to revisit a standardized enhanced recovery pathway? Am J Surg 208:650-655

22. Baraza W, Wild J, Barber W, Brown S (2010) Postoperative management after loop ileostomy closure: are we keeping patients in hospital too long? Ann R Coll Surg Eng1 92:51-55

23. Bliss LA, Maguire LH, Chau $Z$ et al (2015) Readmission after resections of the colon and rectum: predictors of a costly and common outcome. Dis Colon Rectum 58:1164-1173

24. Kirchhoff P, Clavien PA, Hahnloser D (2010) Complications in colorectal surgery: risk factors and preventive strategies. Patient Saf Surg 4:5

25. Evans HL, Lober WB (2017) A pilot use of patient-generated wound data to improve postdischarge surgical site infection monitoring. JAMA Surg 152:595-596

26. Lober WB, Evans HL (2019) Patient-generated health data in surgical site infection: changing clinical workflow and care delivery. Surg Infect (Larchmt) 20:571-576

27. Barberan-Garcia A, Ubré M, Roca J et al (2018) Personalised prehabilitation in high-risk patients undergoing elective major abdominal surgery: a randomized blinded controlled trial. Ann Surg 267:50-56

28. Barberan-Garcia A, Ubre M, Pascual-Argente N et al (2019) Postdischarge impact and cost-consequence analysis of prehabilitation in high-risk patients undergoing major abdominal surgery: secondary results from a randomised controlled trial. Br J Anaesth 123:450-456

29. Keller DS, Khorgami Z, Swendseid B, Khan S, Delaney CP (2014) Identifying causes for high readmission rates after stoma reversal. Surg Endosc 28:1263-1268. https://doi.org/10.1007/s0046 4-013-3320-x

30. Hamidi M, Hanna K, Omesiete P et al (2019) Does it matter where you get your surgery for colorectal cancer. Int J Colorect Dis 34:2121-2127

31. Aquina CT, Probst CP, Becerra AZ et al (2016) High volume improves outcomes: the argument for centralization of rectal cancer surgery. Surgery 159:736-748

32. Ananthakrishnan AN, McGinley EL, Binion DG (2008) Does it matter where you are hospitalized for inflammatory bowel disease? A nationwide analysis of hospital volume. Am J Gastroenterol 103:2789-2798

33. Lemini R, Spaulding AC, Osagiede O et al (2019) Disparities in elective surgery for diverticulitis: identifying the gap in care. Am J Surg 218:899-906

34. Bhalla A, Peacock O, Tierney GM et al (2015) Day-case closure of ileostomy: feasible, safe and efficient. Colorect Dis 17(9):820-823

35. Bilimoria KY, Liu Y, Paruch JL et al (2013) Development and evaluation of the universal ACS NSQIP surgical risk calculator: a decision aid and informed consent tool for patients and surgeons. J Am Coll Surg 217(833-42):e1

Publisher's Note Springer Nature remains neutral with regard to jurisdictional claims in published maps and institutional affiliations. 


\section{Affiliations}

\section{T. R. de Paula ${ }^{1}$ S. Nemeth ${ }^{2} \cdot$ R. P. Kiran ${ }^{1}$ - D. S. Keller ${ }^{1,3}$}

T. R. de Paula

tr2576@cumc.columbia.edu

S. Nemeth

skn2115@cumc.columbia.edu

R. P. Kiran

rpk2118@cumc.columbia.edu

1 Division of Colorectal Surgery, Department of Surgery,

Columbia University Medical Center, New York, NY, USA
2 Columbia HeartSource, Department of Surgery, Center for Innovation and Outcomes Research, Columbia University Medical Center, New York, NY, USA

3 Division of Colon and Rectal Surgery, Department of Surgery, Herbert Irving Comprehensive Cancer Center, NewYork-Presbyterian, Columbia University Medical Center, Herbert Irving Pavilion, 161 Fort Washington Avenue, 8th Floor, New York, NY 10032, USA 